\title{
Search for Multi-Coincidence Cosmic Ray Events over Large Distances with the EEE MRPC Telescopes
}

\author{
Marcello Abbrescia ${ }^{1,2}$, Carlo Avanzini ${ }^{3}$, Luca Baldini ${ }^{3,4}$, Rinaldo Baldini Ferroli ${ }^{5}$, Giovanni Batignani ${ }^{3,4}$, \\ Marco Battaglieri ${ }^{6,7}$, Stefano Boi ${ }^{8,9}$, Edoardo Bossini ${ }^{3,4}$, Francesca Carnesecchi ${ }^{10,11}$, Corrado Cicalò ${ }^{9}$, \\ Luisa Cifarelli 10,11, Fabrizio Coccetti ${ }^{12}$, Eugenio Coccia ${ }^{13}$, Alessandro Corvaglia ${ }^{14}$, Daniele De Gruttola ${ }^{15,16}$, \\ Salvatore De Pasquale 15,16, Franco Fabbri 5, Lorenzo Galante 17,18, Marco Garbini 10,12, Gianluca Gemme ${ }^{6}$, \\ Ivan Gnesi 12,19, Stefano Grazzi ${ }^{6,20}$, Despina Hatzifotiadou 10,21, Paola La Rocca 22,23 (D), Zhang Liu 24 , \\ Giuseppe Mandaglio 20,23 (D), Gaetano Maron ${ }^{25}$, Mario Nicola Mazziotta ${ }^{2}$ (D), Alice Mulliri ${ }^{8,9}$, Rosario Nania ${ }^{10}$, \\ Francesco Noferini ${ }^{10}{ }^{(D)}$, Francesco Nozzoli ${ }^{26}$, Federico Palmonari ${ }^{10,11}{ }^{\mathbb{D}}$, Marco Panareo ${ }^{14,27}$ (D), \\ Maria Paola Panetta 12,14, Riccardo Paoletti ${ }^{3,28}$, Carmelo Pellegrino ${ }^{25}$, Ombretta Pinazza ${ }^{10}$, Chiara Pinto ${ }^{22,23}$, \\ Silvia Pisano ${ }^{5,12}$, Francesco Riggi ${ }^{22,23, *}$, Giancarlo Righini ${ }^{29}$ (D), Cristina Ripoli 15,16, Matteo Rizzi ${ }^{2}$, \\ Gabriella Sartorelli 10,11, Eugenio Scapparone ${ }^{10}$, Marco Schioppa ${ }^{19,30}$, Angelo Scribano ${ }^{28}$, Marco Selvi 10 , \\ Gabriella Serri ${ }^{8,9}$, Sandro Squarcia 6,31, Marco Taiuti 6,31, Giuseppe Terreni ${ }^{3}$, Antonio Trifirò 20,23, \\ Marina Trimarchi ${ }^{20,23}$, Cristina Vistoli ${ }^{25}$, Lucia Votano ${ }^{32}$, Crispin Williams 10,24, Antonino Zichichi 10,11,21 \\ and Roman Zuyeuski 24
}

check for updates

Citation: Abbrescia, M.; Avanzini, C.; Baldini, L.; Baldini Ferroli, R.; Batignani, G.; Battaglieri, M.; Boi, S.; Bossini, E.; Carnesecchi, F.; Cicalò, C.; et al. Search for Multi-Coincidence Cosmic Ray Events over Large Distances with the EEE MRPC Telescopes. J 2021, 4, 838-848. https://doi.org/10.3390/j4040057

Academic Editor: Huirong Yan

Received: 29 October 2021

Accepted: 24 November 2021

Published: 1 December 2021

Publisher's Note: MDPI stays neutral with regard to jurisdictional claims in published maps and institutional affiliations.

Copyright: (C) 2021 by the authors. Licensee MDPI, Basel, Switzerland. This article is an open access article distributed under the terms and conditions of the Creative Commons Attribution (CC BY) license (https:/ / creativecommons.org/licenses/by/ $4.0 /)$.
Dipartimento Interateneo di Fisica, Università di Bari, 70125 Bari, Italy; Marcello.Abbrescia@ba.infn.it INFN, Sezione di Bari, 70125 Bari, Italy; Mazziotta@ba.infn.it (M.N.M.); rizzi@ba.infn.it (M.R.)

INFN, Sezione di Pisa, 56127 Pisa, Italy; Avanzini@pi.infn.it (C.A.); luca.baldini@pi.infn.it (L.B.); Batignani@pi.infn.it (G.B.); Bossini@pi.infn.it (E.B.); riccardo.paoletti@pi.infn.it (R.P.); terreni@pi.infn.it (G.T.) 4 Dipartimento di Fisica, Università di Pisa, 56127 Pisa, Italy

INFN, Laboratori Nazionali di Frascati, 00044 Frascati, Italy; Baldini@lnf.infn.it (R.B.F.); Fabbri@lnf.infn.it (F.F.); Pisano@lnf.infn.it (S.P.)

6 INFN, Sezione di Genova, 16146 Genova, Italy; battaglieri@ge.infn.it (M.B.); Gemme@ge.infn.it (G.G.); Grazzi@ge.infn.it (S.G.); Squarcia@ge.infn.it (S.S.); Marco.Taiuti@ge.infn.it (M.T.)

7 Thomas Jefferson National Accelerator Facility, Newport News, VA 23606, USA

8 Dipartimento di Fisica, Università di Cagliari, 09124 Cagliari, Italy; Boi@ca.infn.it (S.B.); Mulliri@ca.infn.it (A.M.); Serri@ca.infn.it (G.S.)

9 INFN, Sezione di Cagliari, 09042 Cagliari, Italy; Cicalo@ca.infn.it

10 INFN, Sezione di Bologna, 40127 Bologna, Italy; Carnesecchi@bo.infn.it (F.C.); Luisa.Cifarelli@bo.infn.it (L.C.); Garbini@bo.infn.it (M.G.); Hatzifotiadou@cern.ch (D.H.); Rosario.Nania@bo.infn.it (R.N.); Noferini@bo.infn.it (F.N.); Palmonari@bo.infn.it (F.P.); Pinazza@bo.infn.it (O.P.); Sartorelli@bo.infn.it (G.S.); Scapparone@bo.infn.it (E.S.); selvi@bo.infn.it (M.S.); crispin.williams@cern.ch (C.W.);

Zichichi@bo.infn.it (A.Z.)

11 Dipartimento di Fisica e Astronomia, Università di Bologna, 40127 Bologna, Italy

12 Centro Ricerche Enrico Fermi, 00184 Roma, Italy; Coccetti@cref.it (F.C.); Gnesi@cref.it (I.G.); Panetta@le.infn.it (M.P.P.)

13 Gran Sasso Science Institute, 67100 L'Aquila, Italy; Coccia@gssi.infn.it

14 INFN, Sezione di Lecce, 73100 Lecce, Italy; Corvaglia@le.infn.it (A.C.); marco.panareo@le.infn.it (M.P.)

15 Dipartimento di Fisica, Università di Salerno, 84084 Salerno, Italy; Degruttola@sa.infn.it (D.D.G.); DePasquale@sa.infn.it (S.D.P.); Ripoli@sa.infn.it (C.R.)

16 INFN, Gruppo Collegato di Salerno, 84084 Salerno, Italy

17 Dipartimento di Scienze Applicate e Tecnologia, Politecnico di Torino, 10129 Torino, Italy; Galante@to.infn.it

18 INFN, Sezione di Torino, 10125 Torino, Italy

19 INFN, Gruppo Collegato di Cosenza, 87036 Cosenza, Italy; Marco.Schioppa@gmail.com

20 Dipartimento di Scienze Matematiche e Informatiche, Scienze Fisiche e Scienze della Terra, Università di Messina, 98166 Messina, Italy; gmandaglio@unime.it (G.M.); atrifiro@unime.it (A.T.); Trimarchi@unime.it (M.T.)

21 CERN, Esplanade des Particules 1, P.O. Box 1211 Geneva, Switzerland

22 Dipartimento di Fisica e Astronomia "E.Majorana", Università di Catania, 95123 Catania, Italy; paola.larocca@ct.infn.it (P.L.R.); Chiara.Pinto@ct.infn.it (C.P.)

23 INFN, Sezione di Catania, 95123 Catania, Italy

24 ICSC World Laboratory, P.O.Box 1211 Geneva, Switzerland; Liu@cern.ch (Z.L.); Roman.Zuyeuski@cern.ch (R.Z.) 
25 INFN, CNAF, 40127 Bologna, Italy; Maron@cnaf.infn.it (G.M.); Pellegrino@bo.infn.it (C.P.); vistoli@cnaf.infn.it (C.V.)

26 INFN, Trento Institute for Fundamental Physics and Applications, 38123 Trento, Italy; Nozzoli@tn.infn.it

27 Dipartimento di Matematica e Fisica, Università del Salento, 73100 Lecce, Italy

28 Dipartimento di Scienze Fisiche, della Terra e dell'Ambiente, Università di Siena, 53100 Siena, Italy; Scribano@si.infn.it

29 CNR Istituto di Fisica Applicata "Nello Carrara", 50019 Sesto Fiorentino, Italy; Righini@ifac.cnr.it

30 Dipartimento di Fisica, Università della Calabria, 87036 Rende, Italy

31 Dipartimento di Fisica, Università di Genova, 16146 Genova, Italy

32 INFN, Laboratori Nazionali del Gran Sasso, 67100 Assergi, Italy; Votano@lnsg.infn.it

* Correspondence: Francesco.Riggi@ct.infn.it

\begin{abstract}
The existence of independent, yet time correlated, Extensive Air Showers (EAS) has been discussed over the past years, with emphasis on possible physical mechanisms that could justify their observation. The detector network of the Extreme Energy Events (EEE) Collaboration, with its approximately 60 cosmic ray telescopes deployed over the Italian territory, has the potential to search for such events, employing different analysis strategies. In this paper, we have analyzed a set of EEE data, corresponding to an approximately five month observation period, searching for multi-coincidence events among several far telescopes, within a time window of $1 \mathrm{~ms}$. Events with up to 12 coincident telescopes have been observed. Results were compared to expectations from a random distribution of events and discussed with reference to the relativistic dust grain hypothesis.
\end{abstract}

Keywords: cosmic rays; dust grains; extensive air showers; EEE experiment; multi-coincidence events

\title{
1. Introduction
}

After travelling, often for millions of years, through the interstellar and intergalactic medium, primary cosmic particles may impact on the top of the Earth atmosphere, creating, through a variety of physical processes, complex structures, which we denote as Extensive Air Showers. Investigation of these showers has become, since their discovery almost one century ago, a powerful strategy to better understand the properties (energy, direction, and mass composition) of the primaries, their origin, and the accelerating mechanisms. Significant progress has been made on each of these aspects over the last decades via the use of large arrays of detectors, able to measure the correlated arrival of different secondary products from the shower.

It has been long debated whether physical mechanisms may exist that are able to produce independent, yet time correlated, showers separated by large distances, possibly hundreds or thousands of kilometers, on the Earth surface (see Figure 1). In such a case, a different strategy to search for multi-shower events must be adopted, because the surface to be covered would not allow the deployment of individual detectors over the entire area of interest with the required granularity. The answer to this question may come, at least partially, from a sparse array of detectors with a reasonable sensitive area, distributed over a large territory. Apart from the question of the very existence of such events, one of the main difficulties in designing a detection setup well suited to this purpose is the present uncertainty regarding their arrival rate and the range of distances where one may expect such correlations.

The mechanisms, which in principle could justify the existence of such exotic events, are still poorly known, and, even in those few cases where it has been possible to arrive at quantitative estimates of the expected rate and of typical separation distances between correlated showers, the uncertainties in the different parameters of the model make such estimates only a very crude guess, by several orders of magnitude.

Two different classes of models may in principle be involved in the production of correlated showers in the Earth's atmosphere. The first class assumes the existence of two primary cosmic rays, which should be correlated from their origin, travelling over very large distances without significantly losing their momentum correlations. This hypothesis 
is actually very difficult to accept, because it would imply primaries travelling at the speed of light and without electric charge (photons or neutrinos), to keep their time correlation and to avoid deflections by magnetic fields along their travel.

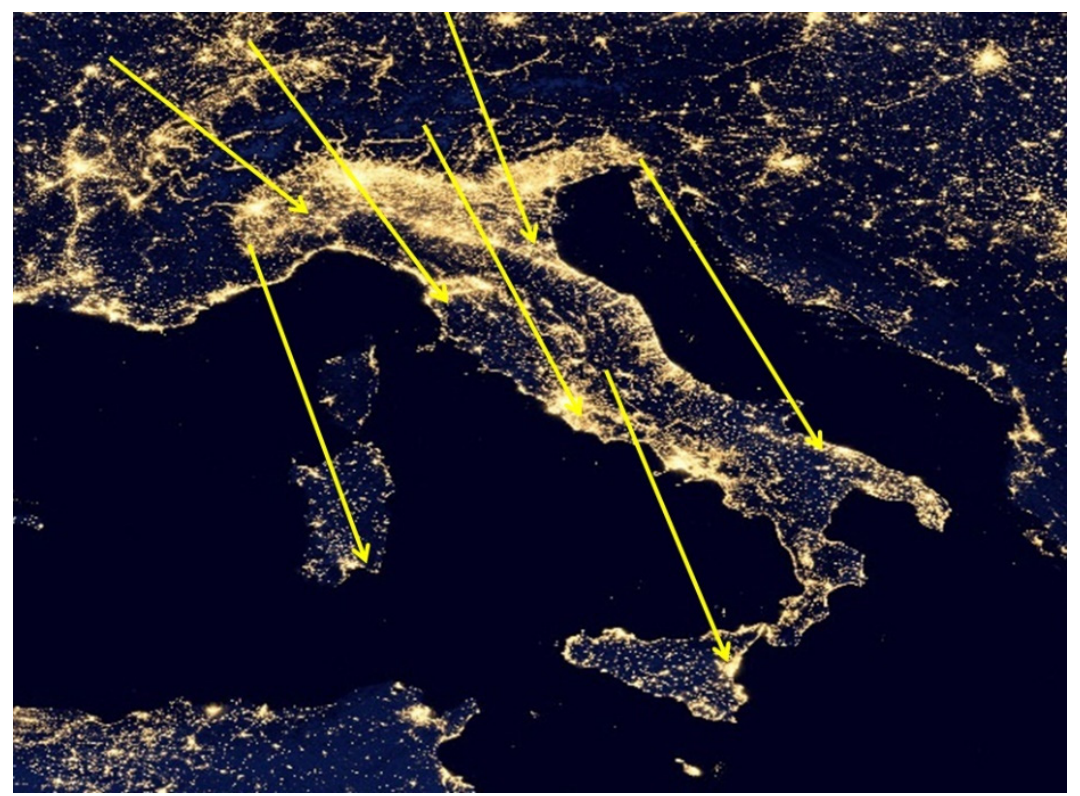

Figure 1. The possible existence of independent air showers arriving at large relative distances may be searched for by a time correlation analysis of cosmic ray data collected by the EEE network over the Italian territory.

The second class is based on the possibility that a single primary particle may locally interact, for instance within the Solar System, producing two or several correlated secondaries, which in turn could be at the origin of independent EASs developing in the Earth atmosphere. This hypothesis seems more promising, because it involves a variety of different possibilities and physical interaction mechanisms that could be worked out to arrive at quantitative estimates, although within a large range.

One such mechanism, and probably the only one that has been largely exploited, is the Gerasimova-Zatsepin (GZ) effect [1], where the photodisintegration of a heavy primary nucleus in the solar radiation field is assumed. It has been shown by different authors [2-10] that this mechanism could predict the production of two correlated showers impinging on the Earth at distances varying between 10 and $1000 \mathrm{~km}$, with estimated rate expectations from $10^{-3}$ to 1 event per $\mathrm{km}^{2}$ per year, depending on the mass and energy of the primary nucleus, and on the influence of the interplanetary magnetic field. Because of these large uncertainties, it is nearly impossible to devise precise experimental conditions to test the existence of such events and to measure their rate, if observed.

Searching for these events has involved, so far, the use of a number of detectors deployed at large distances and taking data for a long time, usually in the context of a wide program of cosmic ray measurements, along with careful analysis strategies to see if anomalous events can be found in the bulk of the collected datasets.

A few attempts to look for any coincidence event between far detectors have revently been carried out [11-15], mostly with scintillator-based detection stations, but no clear positive evidence has been reported until now. More recently, the EEE Collaboration, which operates a network of approximately 60 cosmic ray telescopes based on Multigap Resistive Plate Chambers, installed in High School locations over the Italian territory, has reported a few candidate events, observed in a period covering several years of data collection. These potential candidates were observed through two different analysis strategies.

The first analysis involved 10 EEE sites, where two telescopes were installed in the same metropolitan area, within a relative distance of $1 \mathrm{~km}$ or less, thus detecting standard 
Extensive Air Showers. All possible combinations between shower events detected in each site were then analyzed within time windows between 1 and a few $10^{-5} \mathrm{~s}$, comparing the number of observed events in each interval to the estimated random background. This strategy allowed the reporting of a few candidate events, with a $p$-value smaller than 0.05 , over a data-collection period of several years $[16,17]$.

A second method was employed, looking at possible coincident events between any pair of telescopes in the network, selecting only multi-track events detected in each telescope in order to reduce the spurious rate and to better select individual shower events. Even in this case, the time window of where to search for any enhancement with respect to the expected random background was progressively reduced, from 1 to a few $10^{-5} \mathrm{~s}$, and a slight excess was found with respect to the estimated background, in a time window of less than $10^{-4} \mathrm{~s}[18]$.

In this paper, we report a preliminary result of a new, different analysis strategy, which searches for multi-coincidence events, i.e., events which simultaneously involve the arrival of particles to several telescopes located at distances larger than the typical size of standard individual air showers (a few $\mathrm{km}$ ). This strategy could be particularly suited to the study of exotic events where several, not merely two, independent showers propagate in the Earth atmosphere, leading to secondary particles being detected at large relative distances. In such a case, a possible physical mechanism able to justify events with this topology would be the arrival of different primaries at the top of the atmosphere, separated by large distances, but with a common origin. This in turn could be related to a "super-primary" object, able to produce time correlated fragments at heights larger than 10-15 km from the ground, but still not too far away from the Earth. In this context, relativistic dust grains [19] have been sometimes considered as potential candidates for such mechanisms.

Some considerations regarding the properties of dust grains and their possible connection to extensive air showers are discussed in Section 2, while Section 3 is devoted to demonstrating the main parameters of the EEE cosmic ray telescopes network employed in the present analysis, along with the running conditions. Section 4 reports the analysis strategy and the results of this investigation. Some concluding remarks and outlook are finally discussed in Section 5 .

\section{Dust Grains and Extensive Air Showers}

Relativistic dust grains have been previously discussed [19] as possible candidates to explain the existence of the largest extensive air showers created in the Earth atmosphere. Cosmic dust grains consist of small particles of solid material dispersed in interstellar space. The size of these particles may vary from just a few molecules to grains with a size of $100 \mu \mathrm{m}$. A typical dust grain, however, contains from $10^{6}$ to $10^{9}$ atoms and has a diameter of $10^{-8}$ to $10^{-7} \mathrm{~m}$ (i.e., 10 to 100 nanometers).

Concerning their composition, they are primarily composed of elements that are abundant in the Universe. After hydrogen and helium, the most abundant elements are oxygen, carbon, and nitrogen. These three elements, along with magnesium, silicon, and iron, could be the most important components of interstellar dust.

Recent results concerning the presence of dust grains in the Solar System were obtained from the Cassini Cosmic Dust Analyzer (CDA) [20], which detected dust particles from $10^{-6} \mathrm{~m}$ to $10^{-9} \mathrm{~m}$, measuring their charge, speed, and composition. Apart from the icy dust typical of the Saturn system, evidence has been found of a different class of interstellar dust, containing calcium, carbon, iron, magnesium, silicon, sulfur, and other materials, which points out the interstellar nature of these particles. It transpired that they were able to traverse the Solar System due to their speed (higher than 70,000 km/h), which was too fast for the Solar System's gravity to capture them.

It has been speculated by several authors that dust grains may attain relativistic energies. A mechanism of statistical acceleration due to the collisions of such particles with magnetized clouds was proposed by Fermi [21]. According to this mechanism, dust grains may achieve energies up to $10^{13}-10^{14} \mathrm{eV}$, which results in speeds above the thermal velocity 
of the medium (suprathermal velocity). After that, the grain moves in a homogeneous magnetic field, $\mathrm{H}_{0}$, and may be further accelerated by the magnetic pumping mechanism, due to field irregularities of strength, $k \mathrm{H}_{0}$, over length scales smaller than the grain Larmor radius [22]. These irregularities produce an increase in momentum, irrespective of the value of $k$. Although the value of $k$ is not known, it has been argued [23] that even a value of the order of 5 would be compatible with observed polarimetric effects.

When a cosmic dust grain enters the atmosphere, it may undergo fragmentation due to the air molecules, producing a beam of energetic ions and nuclei. Nuclear interactions start to be relevant at heights of the order of $50 \mathrm{~km}$ from the Earth. Most of the EASs detected at the Earth originate from nuclei that interact at heights in the order of 10-15 km from the Earth. Previous speculations concerning dust grains fragmenting in the region $50-100 \mathrm{~km}$ from the Earth considered the possibility of producing many small showers as the result of the interaction of the fragmentation products. The overall effect of these multiple showers would be, however, a single shower much wider than the usual showers initiated by single nuclei. Although showers with sizes exceeding a few $\mathrm{km}$ have not been observed so far, this also depends on the typical structure of the arrays being used to reconstruct them. Presently, only the Pierre Auger Observatory [24] and the Telescope Array [25], with typical sizes in the order of a few $10^{3} \mathrm{~km}^{2}$, have the potential to observe correlated particles at distances in the order of 10-50 km, depending on appropriate choices of the detection trigger, so the existence of overlapping showers that could mimic a single shower of even larger dimensions cannot be ruled out.

It must be considered that small amounts of air molecules are still present at heights larger than $100 \mathrm{~km}$, which conventionally marks the border of space from the atmosphere. As an example, while $90 \%$ of the atmospheric mass lies within $16 \mathrm{~km}$ of the sea level, approximately $3 \times 10^{-7}$ of this mass is presently outside $100 \mathrm{~km}$, and satellites may experience a non-negligible atmospheric drag, even at altitudes exceeding $500 \mathrm{~km}$. This may justify possible interactions and the consequent disruption of dust grains, even at distances much larger than previously considered, with a diverging beam of secondary particles producing, in turn, energetic showers propagating in the Earth atmosphere as independent cascades. While, at present, no detailed calculation exists regarding this interaction process and the subsequent formation of independent, yet correlated, extensive air showers, with an estimation of the expected abundance of such events, we may at least qualitatively consider that several independent showers could develop, even at large relative distances, of the order of several hundred $\mathrm{km}$. Even though such events have not been observed so far, the EEE network has the capability to quantitatively investigate the possible existence of such events, or to set an upper limit to their abundance, due to its sparse configuration over a large territory, its high duty cycle, and overall time exposure.

\section{The EEE Network}

The EEE Collaboration designed, more than 15 years ago, an array of charged-particle tracking telescopes, based on Multigap Resistive Plate Chambers (MRPC), to be employed within educational and scientific initiatives. Currently, approximately 60 telescopes have been built and installed across Italy and CERN, mostly inside high school buildings, with a direct involvement of students and teachers in the construction, installation, maintenance, and data monitoring. Apart from a few towns, which host two or more telescopes in the same area, the separation distance between individual telescopes exceeds the size of individual showers, allowing for the study of potential long-distance correlations.

The distribution of all the accessible distances between any two telescopes is shown in Figure 2. The peaks at very small distances correspond to those telescope pairs located in the same town (relative distances smaller than $3 \mathrm{~km}$ ), which will be excluded from the present analysis. Apart from that, a broad distribution is evident, which covers from approximately $20 \mathrm{~km}$ to more than $1200 \mathrm{~km}$. 


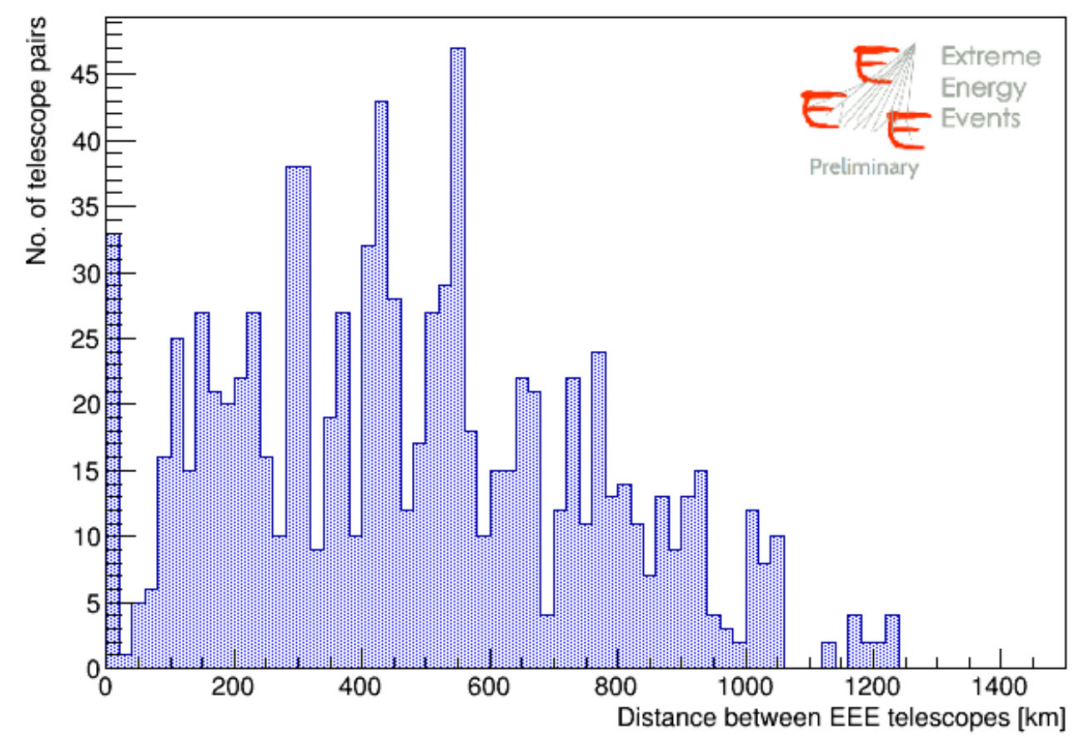

Figure 2. Distribution of the distances between two telescopes within the EEE network. The peaks at very small distances correspond to those telescope pairs located within the same metropolitan area. All the other pairs exhibit a broad distribution, covering relative distances between approximately $20 \mathrm{~km}$ and more than $1200 \mathrm{~km}$.

The EEE network and the performance of the individual detectors have been described in many previous papers [26,27]. Here we will only briefly recall its main features and working parameters.

A typical EEE telescope consists of three large area (approximately $160 \times 82 \mathrm{~cm}^{2}$ ) MRPCs. Each MRPC has six gas gaps obtained by a sandwich of seven thin glass plates spaced apart by $300 \mu \mathrm{m}$, with a continuous flow $(1-2 \mathrm{~L} / \mathrm{h})$ of a gas mixture of $\mathrm{C}_{2} \mathrm{H}_{2} \mathrm{~F}_{4}$ $(98 \%)$ and $\mathrm{SF}_{6}(2 \%)$. A high voltage to the chambers, in the range $18-20 \mathrm{kV}$, is provided by DC/DC converters. To measure the hit coordinates, the detector is segmented with 24 copper strips $(2.5 \mathrm{~cm}$ wide, with $0.7 \mathrm{~cm}$ pitch), giving the transverse coordinate, while the other (longitudinal) coordinate is given by the time difference between the signals arriving at the two strip ends, measured by TDCs, for an overall number of channels of 144. Spatial resolution is of the order of $1 \mathrm{~cm}$ and $1.5 \mathrm{~cm}$ on the transverse and longitudinal coordinates, respectively. The average time resolution on the time-of-flight between the chambers is 240 ps. Most of the MRPCs (77\%) have a detection efficiency higher than $90 \%$.

Data acquisition and control is carried out by a LabView software, with the possibility to remotely control most of the telescope operations. For the purpose of synchronizing the events measured by each telescope, the various stations are equipped with a GPS unit, stamping in time the events with a resolution of about $40 \mathrm{~ns}$.

Tracking of the charged particles traversing the telescope is achieved by reconstruction of the hits on each chamber, merging the hits into clusters, and then fitting the various cluster combinations with straight lines. Multi-track events, although with a low rate (0.1-1\% with respect to single-track events), are also observed and analyzed.

Different analyses of data collected by the EEE network have been carried out over the past years [16-18,28-31], including measurement of the local cosmic ray flux, the study of Forbush variations, the search for anisotropies, and the detection of Extensive Air Showers. The search for long distance correlations is one of the main physics topics of interest for the EEE Collaboration, as discussed in the Introduction.

\section{Running Conditions and Analysis Strategy}

A dataset collected by the EEE network over a period of several months (October 2018-February 2019) was considered in the present analysis. All collected data from the different telescopes were processed on a day-by-day basis to build daily spectra of 
the number of telescopes in coincidence within a time interval of $1 \mathrm{~ms}$. No cut on the relative orientation between the tracks detected in different telescopes was assumed to select the events. All spectra were then summed to get the number of events as a function of the number of telescopes in coincidence within $1 \mathrm{~ms}$. A minimum bias requirement was introduced to select the events, namely, reliable GPS information and at least one good track reconstructed in each telescope. Over a period of approximately 5 months, apart from a few days for maintenance or hardware problems, the average number of operational telescopes was approximately 30 . However, because this number differed from day to day, as shown in Figure 3, the actual number of operational telescopes in each day was taken into account to estimate the spurious coincidence rate.

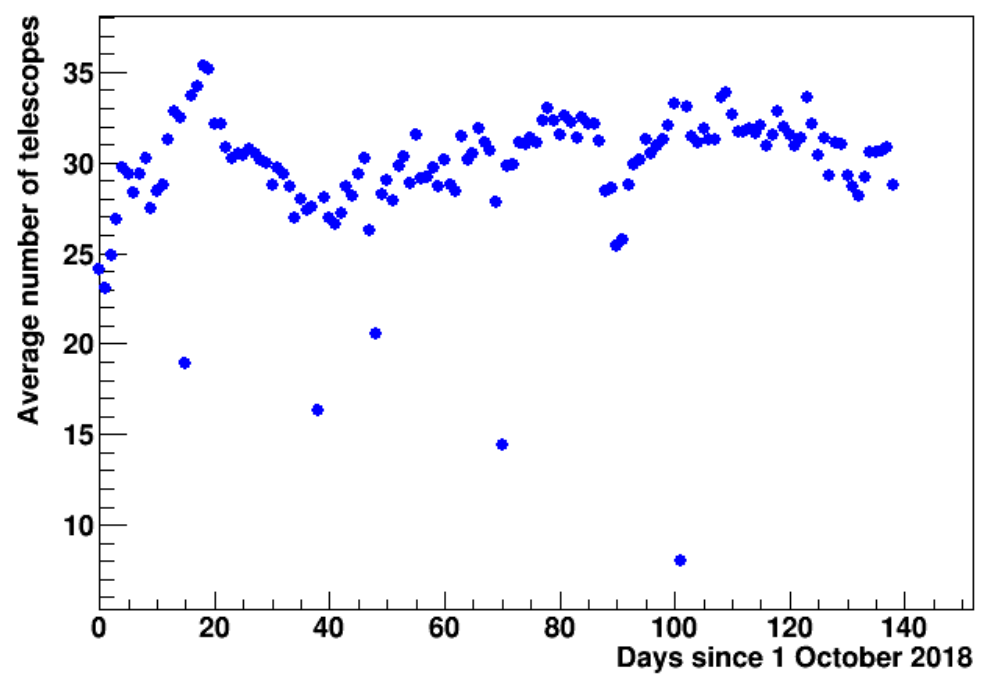

Figure 3. Average number of EEE operational telescopes over the period October 2018-February 2019. Spurious coincidence rate was evaluated on a daily basis, taking into account the effective number of working telescopes.

Searching for events that involve $k$ telescopes within a time interval, $\Delta t$, it is important to evaluate the number of expected spurious events, to compare the two distributions, and evaluate the significance of a $k$-fold coincidence event.

The spurious rate $R_{\text {spurious }}$ for a specific combination of $k$ telescopes may be estimated by

$$
\mathrm{R}_{\text {spurious }} \sim \mathrm{k}\left(\mathrm{R}_{\text {single }}\right)^{\mathrm{k}} \Delta \mathrm{t}^{\mathrm{k}-1}
$$

where $R_{\text {single }}$ is the average rate of each individual telescope, which in our dataset was about $29 \mathrm{~Hz}$. As an example, the estimated spurious rate for a specific combination of 3 telescopes would be about $0.073 \mathrm{~Hz}$, equivalent to 6300 spurious events/day within a time window of $1 \mathrm{~ms}$, compatible with the typical site-to-site distances at the speed of light. This means that no meaning could be associated to a mere coincidence between 3 detectors with such a large value of the coincidence time window.

Of course, for an increasing number of fired telescopes in the same event, the estimated rate of random events will rapidly decrease, and for $n=10$, it can be estimated to be $4 \times 10^{-12} \mathrm{~Hz}$. However, looking for any multiple coincidence between a large number of detectors, we have to consider the number of possible combinations of $k$ telescopes over an overall number, $n$, of working telescopes, provided by:

$$
C_{n, k}=\left(\begin{array}{c}
n \\
k
\end{array}\right)=\frac{n !}{k !(n-k) !}=\frac{1}{k !} \prod_{i=0}^{k-1}(n-i)
$$

which increases rapidly with $k$ and $n$. 
Figure 4 shows, as blue squares, the expected spurious rate (within a time window of $1 \mathrm{~ms}$ ) for any specific combination of $k$ telescopes out of an overall number $n=30$. The number of combinations of $k$ telescopes is shown in the same plot with red symbols. It increases up to $k=n / 2$, then decreases again. The overall spurious rate, arising from any coincident event involving $k$ telescopes, is also plotted in Figure 4 as green triangles. These values can be taken as an estimate of the coincidence rate, which can be expected when a $k$-fold coincidence is observed with a number of operational telescopes of 30 .

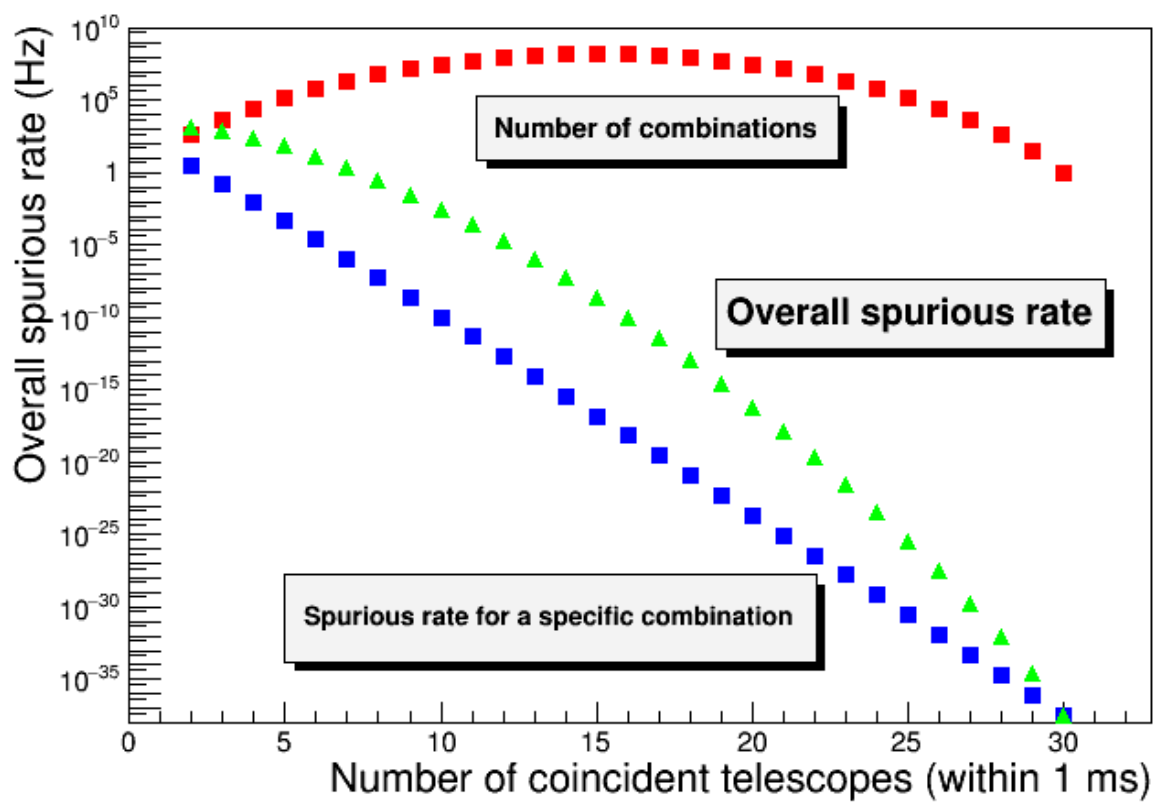

Figure 4. Estimation of the expected spurious rate between $k$ telescopes out of a total number of $n=30$ operational telescopes within a time interval of $1 \mathrm{~ms}$ and assuming an average rate of $29 \mathrm{~Hz}$ for the individual telescopes. Blue symbols show how the spurious rate scales for a specific combination of $k$ telescopes, while the red symbols give the number of combinations of $k$ telescopes out of $n=30$. The overall spurious rate, for any combination of $k$ telescopes, is also plotted (green triangles).

\section{Results}

The number of coincident events arising from a $k$-fold coincidence between $k$, far telescopes (relative distance larger than $5 \mathrm{~km}$ ), is shown in Figure 5. The statistics refer to a data taking period of about 5 months. With these statistics, a few events with up to 12 telescopes, detecting a muon track within an interval of $1 \mathrm{~ms}$, were even observed. The probability to observe an event with $k$ coincident telescopes roughly scales by an order of magnitude for each telescope added. This means that, for our experimental setup and running conditions, the possibility to observe events with an even larger number of telescopes is strongly limited by the statistics. As an example, a factor 10 in data collection (from 5 months to about 4 years) would be required to observe a few events involving at least 13 telescopes.

Concerning the significance of this distribution, we carried out an analysis of the expected number of spurious events in the same conditions, i.e., with an average counting rate of each telescope as extracted from the data and taking into account the effective number of operational telescopes on a day-by-day basis over all the 5 months period.

To compare the experimental distribution with the expected random trend, we plotted the ratio between the two distributions, as a function of the number of telescopes, in Figure 6. As it can be seen, the experimentally observed distribution is compatible with the expected trend of spurious coincidences, showing no excess of observed events, at least for a number of telescopes, of the order of 10 or smaller. A very slight excess was observed for $k=11$ and 12 , although the ratio is still compatible with unity within the statistical error. This allows the estimation of an upper limit, in our conditions, of a few events per year. 


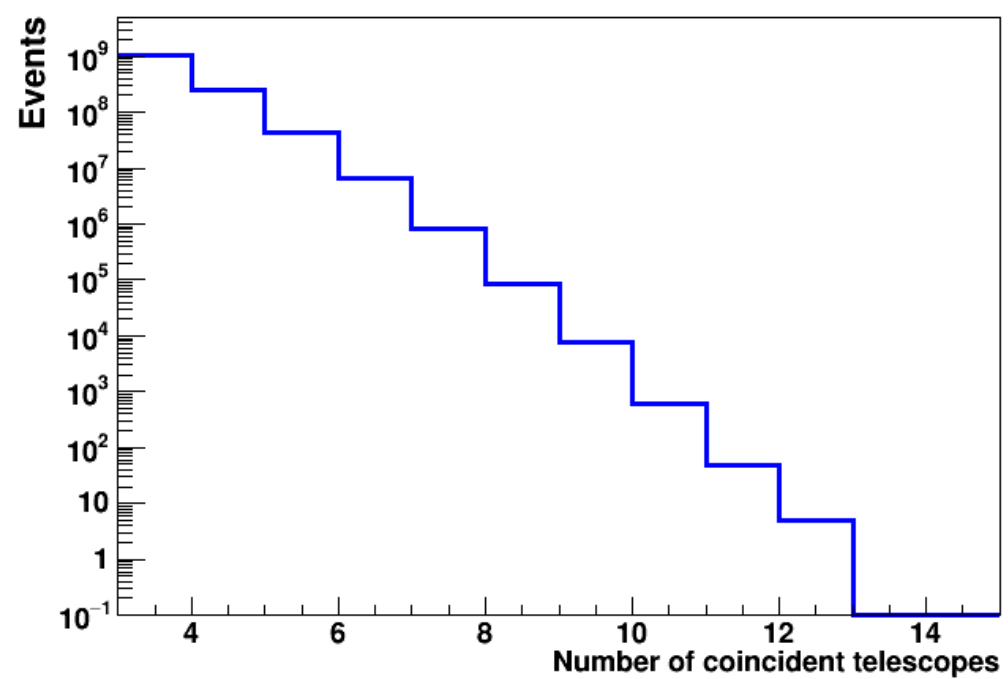

Figure 5. Experimental distribution showing the number of observed coincident events (within a time window of $1 \mathrm{~ms}$ ) between $k$ telescopes, as a function of $k$. A few events involving up to 12 telescopes were observed, with roughly a scale factor of 10 for each additional telescope considered in the coincidence.

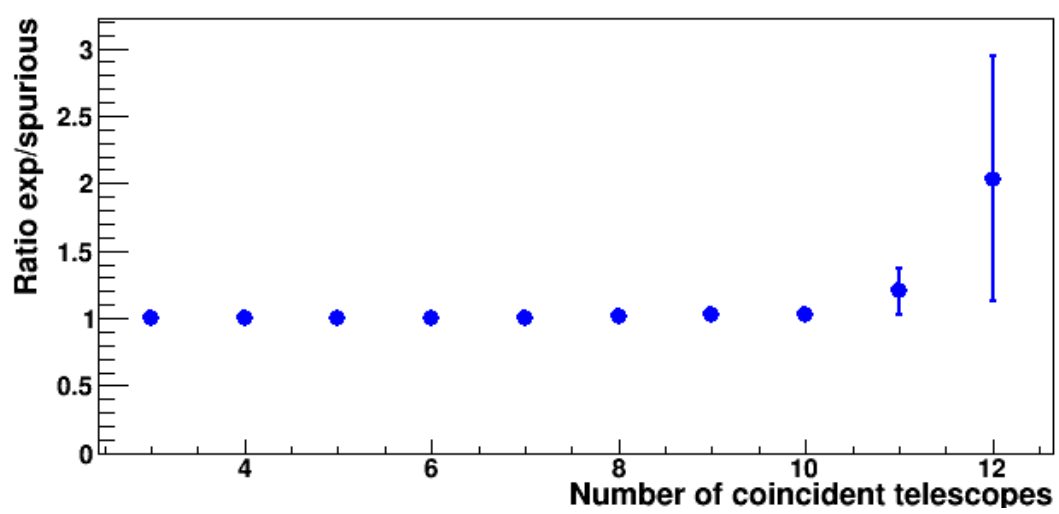

Figure 6. The ratio between experimental and spurious expected distributions of the number of observed coincident events (within a time window of $1 \mathrm{~ms}$ ) between $k$ telescopes is reported as a function of $k$.

Some attempt was made to check whether the observed events with a large number of coincident telescopes (11 and 12) were associated either to peculiar observation days or to a particular range of distances between the sites, but apparently they are distributed more or less randomly in time and with no preference for geographical distributions, which is compatible with observing spurious events.

\section{Conclusions}

A preliminary analysis of a reasonably significant dataset of events collected by the EEE network has been carried out to search for anomalous events involving a large number of telescopes deployed at large separation distances over the Italian territory. The simultaneous arrival of secondary cosmic particles to different telescopes, within a time window of $1 \mathrm{~ms}$, was searched for. The experimental distribution of the collected events, as a function of the number of coincident telescopes, was extracted and compared with the expectation for the random background, as estimated by the average rate of the telescopes and the number of operational sites on a day-by-day basis, over a period of 5 months. With the present collected statistics, events with up to 12 coincident telescopes were observed. The experimental distribution was seen to be in general agreement with 
the expected random contribution. A slight excess was only observed for the events involving the largest number of telescopes $(>10)$, although it was still compatible with a null contribution.

The results allow a very rough estimation of the abundance of such events, which could be, in our current running conditions, less than a few events per year. This is merely a very crude estimate, because many factors, especially the relative distances between the observation sites, may contribute to the possibility to detect multi-coincidence events over large distances. The topology of the EEE network is particularly suited to carry out a search in this direction, because it covers a large range of separation distances with a few dozen stations and a broad distribution of the distances, thus making no a priori selection on the distance of interest, as happens in any experimental setup based on a few detectors deployed in a regular pattern.

The ratio between the number of events involving $k$ and $k+1$ detector stations was found to be of the order of 10 . Because, in our dataset (about 5 months), a few events with $k=12$ were observed, roughly a factor 10 in statistics, in the data collection time, is required to detect events with $k=13$. This is within the present potential of the EEE network, due to the statistics collected over several years and an expected larger average number of operational telescopes, from 30 to approximately 50. A further step may then be planned to extend the present analysis, which will involve additionally checking for the existence of potential multi-coincidence candidate events in a larger dataset or setting a new lower limit to their abundance.

Author Contributions: Conceptualization, M.A., M.B., C.C., L.C., P.L.R., M.N.M., R.N., F.N. (Francesco Noferini), F.R., G.S. (Gabriella Sartorelli) and A.Z.; methodology, M.A., L.C., P.L.R., M.N.M., R.N., F.N. (Francesco Noferini), F.R., G.S. (Gabriella Sartorelli), and A.Z.; software, F.C. (Francesca Carnesecchi), A.C., D.D.G., L.G., M.G., I.G., S.G., P.L.R., G.M. (Giuseppe Mandaglio), M.N.M., F.N. (Francesco Noferini), F.N. (Francesco Nozzoli), M.P.P., C.P. (Carmelo Pellegrino), C.P. (Chiara Pinto), M.R., F.R., M.S. (Marco Schioppa), G.T., C.V. and R.Z.; validation, L.C., R.N., F.N. (Francesco Noferini), F.P. and P.L.R.; formal analysis, investigation, M.A., C.A., L.B., S.B., E.B., C.C., F.C. (Fabrizio Coccetti), D.D.G., M.G., I.G., S.G., D.H., P.L.R., A.M., Z.L., G.M. (Gaetano Maron), M.N.M., A.M., F.N. (Francesco Noferini), M.P., M.P.P., C.P. (Carmelo Pellegrino), O.P., R.P., S.P., F.R., C.R., M.S. (Marco Selvi), G.S. (Gabriella Serri), A.T., M.T. (Marco Taiuti), C.W. and R.Z.; resources, R.B.F., G.B., L.C., E.C., F.F., G.G., D.H., R.N., F.P., R.P., G.R., E.S., A.S., S.D.P., S.S., M.T. (Marina Trimarchi), L.V. and A.Z.; data curation, F.N. (Francesco Noferini); writing-original draft preparation, F.R.; writing-review and editing, M.A., L.C., P.L.R., R.N. and F.R.; visualization, supervision, project administration, M.G. and F.N. (Francesco Noferini); funding acquisition, L.C., M.G., R.N. and F.N. (Francesco Noferini). All authors have read and agreed to the published version of the manuscript.

Funding: This research was funded by Centro Ricerche Enrico Fermi (CREF) and Istituto Nazionale di Fisica Nucleare (INFN).

Acknowledgments: The EEE Collaboration warmly acknowledges the contributions from all high school and teachers who helped in the construction, installation, and monitoring of the MRPC telescopes over the past years.

Conflicts of Interest: The authors declare no conflict of interest.

\section{References}

1. Gerasimova, N.; Zatsepin, G. Disintegration of cosmic ray nuclei by solar photons. Sov. Phys. JETP 1960, 11, 899.

2. Puget, J.; Stecker, F.; Bredekamp, J. Photonuclear interactions of ultrahigh energy cosmic rays and their astrophysical consequences. Astrophys. J. 1976, 205, 638. [CrossRef]

3. Medina-Tanco, G.; Watson, A. The photodisintegration of cosmic ray nuclei by solar photons: The Gerasimova-Zatsepin effect revisited. Astropart. Phys. 1999, 10, 157. [CrossRef]

4. Mollerach, S.; Epele, L.; Roulet, E. On the disintegration of cosmic ray nuclei by solar photons. J. High Energy Phys. $1999,3,17$.

5. Stecker, F.; Salamon, M. Photodisintegration of Ultrahigh Energy Cosmic Rays: A New Determination. Astrophys. J. 1999, 512, 521. [CrossRef]

6. Proceedings of the 29th ICRC Conference, Pune, India, 3-10 August 2005. Available online: https://www.icrr.u-tokyo.ac.jp/can/ icrc2005/ (accessed on 24 November 2021). 
7. La Rocca, P.; Riggi, F. Nuclear Photodisintegration in the Solar Field: Numerical Simulations of the Gerasimova-Zatsepin Effect; Report INFN/AE-08/01; Istituto Nazionale di Fisica Nucleare: Roma, Italy, 2008. [CrossRef]

8. Lafebre, S.; Falcke, H.; Hörandel, J.; Kuijpers, J. Prospects for direct cosmic ray mass measurements through the GerasimovaZatsepin effect. Astron. Astrophys. 2008, 485, 1. [CrossRef]

9. Andersen, K.; Klein, S. High energy cosmic-ray interactions with particles from the sun. Phys. Rev. D 2011, 83, 103519. [CrossRef]

10. van Eijden, J.V.R.; de Jong, S.J.; Timmermans, C.J.W.P. Cosmic ray interactions in the solar system: The Gerasimova-Zatsepin effect. arXiv 2016, arXiv:astro-ph/1606.07693v2.

11. Ochi, N.; Iyono, A.; Kimura, H.; Konishi, T.; Nakamura, T.; Nakatsuka, T.; Ohara, S.; Ohmori, N.; Okei, K.; Saitoh, K.; et al. Search for large-scale coincidences in network observation of cosmic ray air showers. J. Phys. G Nucl. Part. Phys. 2003, $29,1169$. [CrossRef]

12. Fujiwara, Y.; Iyono, A.; Tada, J.; Okei, K.; Ochi, N.; Konishi, T.; Nakatsuka, T.; Ohara, S.; Takahashi, N.; Tokiwa, M.; et al. Search for Simultaneous Parallel EAS Events in Long Baseline EAS Arrays with LAAS. Nucl. Phys. B (Proc. Suppl.) 2006, 151, 481-484. [CrossRef]

13. Iyono, A.; Matsumoto, H.; Okei, K.; Tsuji, S.; Ohara, S.; Ochi, N.; Konishi, T.; Takahashi, N.; Yamamoto, I.; Nakatsuka, T.; et al. Cosmic ray composition studies through the Gerasimova-Zatsepin effects of heavy nuclei at LAAS. Astrophys. Space Sci. Trans. 2011, 7, 327-333. [CrossRef]

14. Smolek, K.; Blaschke, F.; Čermák, J.; Lichard, P.; Pinfold, J.; Pospíšil, P.; Přidal, P.; Smejkal, J.; Soluk, R.; Štekl, I.; et al. ALTA/CZELTA-A sparse very large air shower array: Overview of the experiment and first results. In Proceedings of the 31 ICRC Conference, Lodz, Poland, 7-15 July 2009. Available online: http://icrc2009.uni.lodz.pl/proc/pdf/icrc1300.pdf (accessed on 24 November 2021).

15. Blaschke, F.; Čermák, J.; Hubík, J.; Lichard, P.; Přidal, P.; Smejkal, J.; Smolek, K.; Štekl, I.; Vícha, V. CZELTA: An overview of the CZECH large area time coincidence array. Astrophys. Space Sci. Trans. 2011, 7, 69-73. [CrossRef]

16. Riggi, F.; Abbrescia, M.; Baldini, L.; Baldin Ferroli, R.; Batignani, G. Time and orientation long-distance correlations between extensive air showers detected by the MRPC telescopes of the EEE project. Il Nuovo Cim. C 2017, 40, 196.

17. Abbrescia, M.; Baldini, L.; Ferroli, R.B.; Batignani, G.; Battaglieri, M.; Boi, S.; Bossini, E.; Carnesecchi, F.; Chiavassa, A.; Cicalo, C.; et al. Search for long distance correlations between extensive air showers detected by the EEE network. Eur. Phys. J. Plus 2018, 133, 34. [CrossRef]

18. La Rocca, P.; Abbrescia, M.; Avanzini, C.; Baldini, L.; Ferroli, R.B.; Batignani, G.; Battaglieri, M.; Boi, S.; Bossini, E.; Carnesecchi, F.; et al. Looking for long-range correlations among the EEE telescopes. In Proceedings of the 37th International Cosmic Ray Conference-PoS(ICRC2021), Berlin, Germany, 12-23 July 2021; p. 292.

19. Herlofson, N. Accelerated dust grains and cosmic ray energies. Tellus 1956, 8, 2. [CrossRef]

20. Srama, R.; Ahrens, T.J.; Altobelli, N.; Auer, S.; Bradley, J.G.; Burton, M.; Dikarev, V.V.; Economou, T.; Fechtig, H.; Görlich, M.; et al. The Cassini Cosmic Dust Analyzer. Space Sci. Rev. 2004, 114, 465-518. [CrossRef]

21. Fermi, E. On the origin of cosmic radiation. Phys. Rev. 1949, 75, 1169. [CrossRef]

22. Alfven, H.; Falthammar, C.G. Cosmic Electrodynamics; Oxford University Press: Oxford, UK, 1963.

23. Mendis, D.A.; Wickramasinghe, N.C. On the acceleration of interstellar grains. Astrophys. J. 1976, 42, L11. [CrossRef]

24. Pierre Auger Observatory Web Site. Available online: https:/ /www.auger.org/ (accessed on 24 November 2021).

25. Telescope Array Web Site. Available online: http:/ / www.telescopearray.org/ (accessed on 24 November 2021).

26. Abbrescia, M.; Avanzini, C.; Baldini, L.; Ferroli, R.B.; Batignani, G.; Battaglieri, M.; Boi, S.; Bossini, E.; Carnesecchi, F.; Chiavassa, A.; et al. The Extreme Energy Events experiment: An overview of the telescope performance. J. Instrum. 2018, 13, P08026. [CrossRef]

27. De Gruttola, D.; Abbrescia, M.; Avanzini, C.; Baldini, L.; Ferroli, R.B.; Batignani, G.; Battaglieri, M.; Boi, S.; Bossini, E.; Carnesecchi, F.; et al. Performance of the Multigap Resistive Plate Chambers of the Extreme Energy Events Project. J. Instrum. 2019, 14, C05022. [CrossRef]

28. Abbrescia, M.; Aiola, S.; Antolini, R.; Avanzini, C.; Ferroli, R.B.; Bencivenni, G.; Bossini, E.; Bressan, E.; Chiavassa, A.; Cicalo, C.; et al. Observation of the February 2011 Forbush decrease by the EEE telescopes. Eur. Phys. J. Plus 2011, 126, 61. [CrossRef]

29. Abbrescia, M.; Agocs, A.; Aiola, S.; Antolini, R.; Avanzini, C.; Ferroli, R.B.; Bencivenni, G.; Bossini, E.; Bressan, E.; Chiavassa, A.; et al. The EEE experiment project: Status and first physics results. Eur. Phys. J. Plus 2013, 128, 62. [CrossRef]

30. Abbrescia, M.; Avanzini, C.; Baldini, L.; Ferroli, R.B.; Batignani, G.; Bencivenni, G.; Bossini, E.; Bressan, E.; Chiavassa, A.; Cicalo, C.; et al. Looking at the sub-TeV sky with cosmic muons detected in the EEE MRPC telescopes. Eur. Phys. J. Plus 2015, $130,187$. [CrossRef]

31. Abbrescia, M.; Alici, A.; An, S.; Antolini, R.; Badalà, A.; Baek, Y.; Ferroli, R.B.; Bencivenni, G.; Blanco, F.; Bressan, E.; et al. First detection of extensive air showers with the EEE experiment. Nuovo Cim. B 2010, 125, 243. 